Check for updates

Cite this: RSC Adv., 2019, 9, 36162

\title{
Plasma fatty acid metabolic profiling coupled with clinical research reveals the risk factors for atherosclerosis development in type 2 diabetes mellitus $\dagger$
}

\begin{abstract}
Qianyu Zhou, ${ }^{a}$ Dabing Ren, ${ }^{\text {ab }}$ Yang Xiao, ${ }^{c}$ Lunzhao Yi (D) *ab and Zhiguang Zhou*c
Many publications have reported that the incidence of atherosclerotic cardiovascular diseases is higher in patients with type 2 diabetes mellitus (T2DM) than in the non-diabetic population; however, until now, the reason has been unclear. In this study, 25 males (25/64, 39.06\%) and 19 females (19/54, 35.19\%) had complications with atherosclerosis after two years. To reveal the risk factors for developing atherosclerosis in patients with T2DM, plasma fatty acid metabolic profiling based on gas chromatography-mass spectrometry was combined with the analysis of clinical biochemical indices. The results of partial least squares-discriminant and canonical correlation analyses suggested that C20:0, C22:6n-3, glycosylated hemoglobin, waist circumference, and waist-to-hip ratio are likely to be closely related to T2DM complicated with atherosclerosis. Metabolomic information is a beneficial supplement to existing clinical indices and is useful in predicting the development of a patient's disease and optimizing the treatment.
\end{abstract}

Received 20th September 2019 Accepted 17th October 2019

DOI: $10.1039 / c 9 r a 07634 d$

rsc.li/rsc-advances
Nevertheless, the successful treatment of this disease using drugs requires perturbing the metabolism without causing defects in other metabolic pathways. To date, several therapeutic drugs such as fenofibrate, ${ }^{\mathbf{1 0}}$ rosiglitazone,${ }^{\mathbf{1 1}}$ metformin,${ }^{\mathbf{1 2}}$ and repaglinide ${ }^{13}$ have been used to treat T2DM. However, in most cases, these drugs maintain their functions only at certain levels for the basic physiological needs instead of normalizing dysregulated metabolic systems.

In 2012, 1.5 million deaths directly caused by diabetes were reported, along with 2.2 million deaths due to cardiovascular diseases, chronic kidney disease, and tuberculosis, which are related to higher-than-optimal blood glucose. ${ }^{9}$ Most of this excess mortality risk is attributed to the atherosclerotic cardiovascular disease. ${ }^{\mathbf{1 4}}$ Unfortunately, patients with T2DM have an increased incidence of the atherosclerotic cardiovascular disease as compared with the non-diabetic population. ${ }^{\mathbf{1 5}}$ The reason may be that the two diseases share many antecedent factors that frequently coexist, which have given rise to the "common soil" hypothesis. This cluster of risk factors includes hypertension ${ }^{16,17}$ and dyslipidemia, ${ }^{18}$ which is characterized by elevated plasma triglyceride levels, low levels of high-density lipoprotein (HDL) cholesterol, and high levels of low-density lipoprotein (LDL). ${ }^{19}$ However, the precise mechanism underlying the development of the atherosclerotic cardiovascular disease has not been fully elucidated.

Currently, biochemical changes in T2DM patients treated with certain drugs have been explored by few long-term studies. ${ }^{20,21}$ For instance, after receiving a 48 week treatment
${ }^{a}$ Faculty of Agriculture and Food, Kunming University of Science and Technology, 65920302

${ }^{b}$ Yunnan Food Safety Research Institute, Kunming University of Science and Technology, Kunming, Yunnan, 650500, China

${ }^{c}$ Diabetes Center, Institute of Metabolism and Endocrinology, Department of Endocrinology, The Second Xiangya Hospital, Central South University, Changsha, Hunan, 410011, China.E-mail: zhouzhiguang@csu.edu.cn

$\dagger$ Electronic supplementary information (ESI) available. See DOI: 10.1039/c9ra07634d 
of a single drug, Bao et al. found that serum metabolites in diabetic subjects were considerably altered. ${ }^{22}$ The alterations included increased levels of valine, maltose, glutamate, urate, butanoate, and long-chain fatty acids (C16:0, C18:1, C18:0, octadecanoate, and arachidonate) and decreased levels of glucuronolactone, lysine, and lactate. Drugs were applied to regulate metabolic activity in patients and sustain physiological functions at certain levels rather than to reverse the dysregulation of metabolic networks.

In this study, we have attempted to reveal the risk factors for developing atherosclerosis (AS) in patients with T2DM. A total of 118 patients with T2DM were enrolled. The blood sugar, blood pressure, and blood lipid levels were controlled through a two-year treatment. Alterations in fatty acid metabolism and clinical indices were evaluated before and after treatment. Plasma fatty acid metabolic profiles obtained by gas chromatography-mass spectrometry (GC-MS) and the information of 15 clinic indices were combined for further data analysis. Partial least squares-discriminant analysis (PLS-DA) and canonical correlation analysis (CCA) were employed for the exploration of the key risk factors and the connection between free fatty acids (FFAs), metabolic patterns, and clinical indices.

\section{Experimental}

\section{Sample collection}

There were 160 T2DM patients enrolled in this work. All patients were newly diagnosed and the disease durations were less than 1 year. During the treatment, we tried to control the patients' blood sugar, blood pressure, and blood lipid levels through medication as previously described..$^{23}$ Patients with a BMI of 25 or more were started on metformin; those with a BMI of less than 25 were started on glipizide. All agents were started on half the maximal doses. Subsequently, the dosages were adjusted to meet the goal of glycated hemoglobin A1c (HbA1c) levels less than 7.0\%. Antihypertensive and lipidlowering medication and dosages were determined according to protocol guidelines. ${ }^{23}$ The goal of blood pressure control is lower than 130/80 mmHg. The goal of lipid control is based on recommendations of the United States Adult Treatment Panel III. ${ }^{24}$ There was no statistically significant difference in the types of drugs used for treatment between patients with and without atherosclerosis during the 2 years of treatment. After the twoyear treatment, only 118 patients were controlled well (fasting plasma glucose (FPG), $6.46 \pm 0.98 \mathrm{mmol} \mathrm{L}^{-1} ; 2$ hour postprandial plasma glucose (2hPG), $8.15 \pm 2.22 \mathrm{mmol} \mathrm{L}^{-1}$; total triglycerides (TG), $1.53 \pm 0.96 \mathrm{mmol} \mathrm{L}^{-1}$; total cholesterol (TC), $4.37 \pm 0.86 \mathrm{mmol} \mathrm{L}^{-1} ; \mathrm{HDL}, 1.32 \pm 0.23 \mathrm{mmol} \mathrm{L}^{-1} ; \mathrm{LDL}, 2.28 \pm$ $0.65 \mathrm{mmol} \mathrm{L}^{-1}$; systolic blood pressure (SBP), $110.74 \pm 12.10$ $\mathrm{mmHg}$; diastolic blood pressure (DBP), $70.14 \pm 7.26 \mathrm{mmHg}$ ). Of the 118 T2DM patients, 64 males and 54 females, with ages ranging from 42 years old to 76 years, were enrolled, and the occurrence of AS and plasma fatty acid metabolic profiling was evaluated in these patients. The intima-media thickness (IMT) of common carotid, femoral, and common iliac arteries on the right side were evaluated by high-resolution B-mode ultrasound (128XP/10 system; Acuson, Mountain View, California, USA).
The measurements of IMT were made at the site of greatest thickness. Plaque has an IMT of $\geq 1.3 \mathrm{~mm}$, or is characterized by a focal protrusion into the lumen with a thickness at least $50 \%$ greater than the adjacent intima-media complex; AS has an IMT of $>1.0 \mathrm{~mm}$ and/or plaque on at least one of the three arteries. ${ }^{25}$

Human plasma samples were collected from the 118 T2DM patients before and after the two-year treatment. Demographic and blood biochemical parameters, such as gender, body mass index (BMI), waist circumference (WC), hip circumference (HC), waist-hip ratio (WHR), FPG, 2hPG, HbA1c, alanine transaminase (ALT), total bilirubin (TBIL), blood urea nitrogen (BUN), creatinine (Cr), TG, TC, HDL, LDL, SBP, and DBP were measured by standard methods and recorded.

This study was performed in accordance with the Declaration of Helsinki of the World Medical Association, and was approved by the Ethics Committee of the Second Xiangya Hospital of Central South University. Written informed consent was obtained from all participants.

\section{Solvents and standards}

Oleic acid (C18:1n-9, >99.0\% purity), 11-octadecenoic acid (C18:1n-7, >99.0\% purity), linolenic acid (C18:3n-3, >99.0\% purity), $\gamma$-linolenic acid (C18:3n-6, >99.0\% purity), linoleic acid (C18:2n-6, >99.0\% purity), palmitoleic acid (C16:1n-7, >99.0\% purity), heptadecanoic acid (C17:0, >99.0\% purity), and heptadecanoic acid methyl ester (C17:0 methyl ester, $>99.0 \%$ purity) were purchased from Sigma (St. Louis, MO, USA). The solution $5 \% \mathrm{H}_{2} \mathrm{SO}_{4} / \mathrm{CH}_{3} \mathrm{OH}$ was freshly prepared by diluting $\mathrm{H}_{2} \mathrm{SO}_{4}$ ( $>98.0 \%$ Purity) by chromatographic-grade methanol, and $0.4 \mathrm{M}$ $\mathrm{NaOH} / \mathrm{CH}_{3} \mathrm{OH}$ was freshly prepared in our laboratory by dissolving reagent-grade $\mathrm{NaOH}$ in methanol.

\section{Sample preparation}

Each collected blood sample was immediately centrifuged at $3000 \times g$ for $10 \mathrm{~min}$ and plasma was stored at $-80{ }^{\circ} \mathrm{C}$ until analysis. Sample preparation procedures have been described previously by Yi et al. ${ }^{26}$ In this paper, we considered only the FFAs in plasma, and esterified fatty acid (EFAs) fractions were not included. Aliquots $(200 \mu \mathrm{L})$ of plasma were spiked with internal standard (I.S.) working solution ( $25 \mu \mathrm{L} \mathrm{C17:0} \mathrm{and} 25 \mu \mathrm{L}$ C17:0 methyl ester), and lipid extraction was carried out using hexane. The methyl esters of EFAs were extracted with hexane after the first esterification reaction, and the hexane phase was removed. Then, the methyl esters of FFAs were extracted with hexane after the second esterification reaction and concentrated under $\mathrm{N}_{2}$ gas. Hexane $(100 \mu \mathrm{L})$ was added to each tube prior to analysis.

\section{Gas chromatography-mass spectrometry}

All GC-MS analyses were performed by a Shimadzu GC2010A (Kyoto, Japan) gas chromatography instrument coupled to a GCMS-QP2010 mass spectrometer (Compaq-Pro Linear data system, class5K software). In the gas chromatographic system, a DB-23 capillary column $(30 \mathrm{~m} \times 0.25 \mathrm{~mm}$ I.D., film thickness $0.25 \mu \mathrm{m}$ ) was used. The GC column temperature was programmed at $70-150{ }^{\circ} \mathrm{C}$ at a rate of $20{ }^{\circ} \mathrm{C} \min ^{-1}, 150-180{ }^{\circ} \mathrm{C}$ at a rate of 
$6{ }^{\circ} \mathrm{C} \mathrm{min} \operatorname{mon}^{-1}, 180-220{ }^{\circ} \mathrm{C}$ at a rate of $20{ }^{\circ} \mathrm{C} \min ^{-1}$, then held for $9 \mathrm{~min}$ at $220{ }^{\circ} \mathrm{C}$. The total GC run time was $20 \mathrm{~min}$. The inlet temperature was kept at $250{ }^{\circ} \mathrm{C}$. The helium carrier gas was used

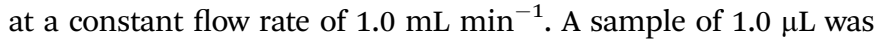
injected at a split ratio of $1: 10$. Mass conditions were as follows: ionization voltage, $70 \mathrm{eV}$; ion source temperature, $200{ }^{\circ} \mathrm{C}$; and full scan mode in the 30-450 $\mu$ mass ranges with $0.2 \mathrm{~s} / \mathrm{scan}$ velocity.

\section{Data analysis}

The structures of the peaks of interest were identified on the basis of the similarity search of the NIST/EPA/NIH Mass Spectral Library (NIST 05) and chemical standards. A total of 21 fatty acids were determined. C16:1n-7, C18:1n-9, C18:1n-7, C18:2n-6, C18:3n-3, and C18:3n-6 were identified according to their retention times, and their mass spectra were compared with corresponding chemical standards, while the others were recognized based on the similarity search of the mass library. For quantification, the internal standard method was employed. For the normalization of the metabolomics data, the metabolites were expressed as the ratio of peak area of corresponding metabolites to that of the internal standard on the same chromatogram.

The input data included 21 fatty acids, total FFAs, and 15 clinical indices. The data matrix was generated for statistical analysis using partial least squares-discriminant analysis (PLSDA), and each of the rows and columns of the matrix represented a sample and variable, respectively. The data matrix was auto-scaled and then analyzed by PLS-DA for the establishment of their classification models. Class membership was predicted by using a discriminant line between two classes obtained by linear discriminant analysis (LDA). The reliability of the classification model was evaluated through 10 -fold cross-validation. The coefficient $\beta$ of the PLS-DA model was introduced to select the key metabolites reflecting the effect of origin. A variable with high coefficient $\beta$ might play an important role in a classification model. ${ }^{27,28}$ The statistical analysis of PLS-DA was performed using the in-house software written in MATLAB (version 6.5, The MathWorks, Natick, MA, USA).

CCA was employed to explore the connection between FFA metabolic patterns and clinical indices. The canonical variables $U$ and $V$ represent the linear combination of clinical indices and the metabolic parameters of fatty acids, respectively. $U_{1}$ and $V_{1}$ were the first pair of canonical variables with a large correlation. The absolute value of the coefficient corresponding to each variable of the first pair of canonical variables reflected the influence of the clinical indices and the metabolic parameters of fatty acids on the variable. The statistical analysis of CCA was performed using the in-house software written in R x64 (version 3.3.1, New Zealand).

\section{Results}

\section{Alterations of T2DM patients' indices after two-year's treatment}

Table 1 lists the 37 indices of the T2DM patients. A total of 26 plasma lipid metabolic indices, namely, the 21 FFAs, total FFAs,
HDL, LDL, TC, and TG, were detected. The other 11 clinical examination indices were BMI, WC, HC, WHR, FPG, 2hPG, HbA1c, ALT, TBIL, BUN, and Cr.

After two years of treatment, most indices decreased significantly (Mann-Whitney $U$ test $p<0.05$ with a signed $T$ value of “1”), except for C22:5n-3, HDL, WHR, and BUN (Mann-Whitney $U$ test, $p>0.05$ with a signed $T$ value of “0”). Statistically, the results indicated that the treatment was effective in downregulating the blood sugar and lipid metabolic levels of T2DM patients. However, WC and HC decreased and WHR was unchanged in the 118 patients.

Two years after the treatment, 25 male T2DM patients $(25 / 64$, $39.06 \%$ ) and 19 female T2DM patients (19/54, 35.19\%) were complicated with AS. Then, 118 T2DM patients were divided into two groups: T2DM and T2DM-AS. In the two groups of patients, all clinical data had no significant difference in baseline (shown in Table 2). After two years, the ALT of T2DM-AS was significantly down-regulated, whereas that of T2DM was not. The Mann-Whitney $U$ test revealed that T2DM and T2DM-AS had significant differences in terms of alterations in ALT, 8 FFAs, and total FFAs. In T2DM-AS, three saturated FFAs and five unsaturated FFAs (C16:0, C18:0, C20:0, C16:1n-9, C18:1n-9, C18:1n-7, C18:2n-6, and C18:3n-6) were down-regulated more significantly than in T2DM. However, the combined effect of these indices was not considered, and only the difference in a single index between the two groups was evaluated.

\section{Index pattern relating to the treatment of T2DM}

The index pattern relating to T2DM before and after treatment was determined through PLS-DA. The information from the 37 indices was used as the input data of PLS-DA for the establishment of a visible model for discriminating 74 T2DM patients without AS before and after treatment. The final optimized two-dimensional (2D) PLS-DA model is presented in Fig. 1(A). T2DM patients before (blue hollow circles) and after (red diamonds) treatment were separated and distinctly grouped with PLS-DA. In addition, the PLS-DA model was evaluated by 10 -fold cross-validation. The area under the receiver operating characteristic curve (AUC) value of 10-fold cross-validation was $96.05 \%$. The total correct rate for the 74 T2DM patients was $91.22 \%$ while the correct rates for these patients before and after two years of treatment were $87.84 \%$ and $94.59 \%$, respectively.

Based on the above PLS-DA model, which has good discrimination and predictive ability, the value of coefficient $\beta$ was used for the evaluation of the importance of these clinical indices and FFA metabolites. The importance of an index increases with the $\beta$ value. Taking AUC and the total correct rate into consideration, five variables of the PLS-DA models were selected as shown in Fig. S1. $\dagger$ In Fig. 1(B), the $\beta$ values of the three clinical indices $(\mathrm{Cr}$, TBIL, and 2hPG) and two FFAs (C20:2n-7 and C20:5n-3) were much higher than those of the other indices. After these five indices were used as input data for the establishment of a new PLS-DA model, the total correct rate and AUC value were $92.22 \%$ and $96.05 \%$, respectively. The screened index pattern was the same as the PLS-DA model of 118 T2DM patients shown in 
Table 1 The clinical data and FFA concentrations for the T2DM patients before and after two years of $\operatorname{treatment}^{a}$

T2DM

Baseline $(n=118) \quad$ Post-treatment $(n=118)$

$$
\begin{aligned}
23.82 & \pm 3.03 \\
83.65 & \pm 7.86 \\
92.81 & \pm 5.97 \\
0.90 & \pm 0.05 \\
6.46 & \pm 0.98 \\
8.15 & \pm 2.22 \\
6.40 & \pm 0.66 \\
21.80 & \pm 10.40 \\
11.66 & \pm 4.44 \\
5.05 & \pm 1.39 \\
80.05 & \pm 14.09 \\
1.53 & \pm 0.96 \\
4.37 & \pm 0.86 \\
1.32 & \pm 0.23 \\
2.28 & \pm 0.65 \\
0.62 & \pm 0.89 \\
4.86 & \pm 2.72 \\
0.96 & \pm 0.48 \\
119.7 & \pm 53.73 \\
2.99 & \pm 1.78 \\
5.61 & \pm 3.93 \\
37.3 & \pm 11.70 \\
120.69 & \pm 58.50 \\
9.50 & \pm 4.60 \\
123.35 & \pm 53.14 \\
1.01 & \pm 0.69 \\
5.10 & \pm 3.76 \\
0.85 & \pm 0.52 \\
1.76 & \pm 1.28 \\
0.95 & \pm 0.49 \\
3.68 & \pm 1.68 \\
18.57 & \pm 6.95 \\
1.44 & \pm 0.85 \\
0.97 & \pm 1.56 \\
1.79 & \pm 2.08 \\
3.75 & \pm 1.90 \\
\hline 66.25 & \pm 191.19
\end{aligned}
$$$$
8.14 \pm 6.68
$$$$
738.86 \pm 251.39
$$

$T$

Variations
C22:6n-3 $\left(\mu \mathrm{mol} \mathrm{L}{ }^{-1}\right)$

Total FFAs $\left(\mu \mathrm{mol} \mathrm{L}^{-1}\right)$

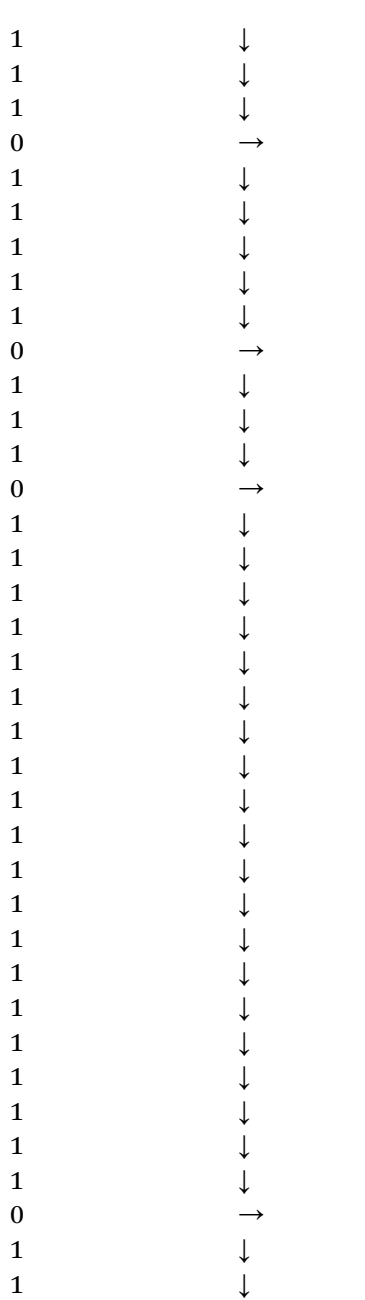

${ }^{a}$ The clinical data are presented as mean \pm SD. $T$ is the Mann-Whitney $U$ test results of T2DM patients before and after two years of treatment; $p$ value of $<0.05$ was considered statistically significant and the signed $T$ value is " 1 ", otherwise " 0 ".

Fig. S2. $\uparrow$ These results indicated that Cr, TBIL, 2hPG, C20:2n-7, and C20:5n-3 are correlated with metabolic disturbances in the therapeutic process of T2DM. After two years of treatment, the $\mathrm{Cr}$, TBIL, 2hPG, C20:2n-7, and C20:5n-3 of all patients were significantly down-regulated, but the two groups (T2DM and T2DM-AS) had no significant difference.

The connection between the clinical indices and FFA metabolic profiles were further explored by analyzing the 15 clinical indices and 21 FFAs concentrations of each of the 74 T2DM patients through CCA. The first pair of typical variables of metabolites $\left(U_{1}\right)$ and clinical indices $\left(V_{1}\right)$ before and after two years of treatment in 74 T2DM patients are shown in Fig. 2. The data show that $R=0.8290$ in Fig. 2(A) while $R=0.8554$ in Fig. 2(D). These results indicate that the FFAs metabolic patterns were closely correlated with the clinical parameters.

The key indices were determined by using the coefficients of the first pair of canonical variables in evaluating the importance of clinical indices and FFAs. The coefficients' absolute values of the first pair of canonical variables in the clinical indices and fatty acids of 74 T2DM patients are shown in Fig. 2. For patients with T2DM before treatment, the most closely related variables between fatty acid metabolism and clinical indices were C18:0, C18:2n-6, C22:6n-3, WC, HC, and WHR. The absolute values of their coefficients were higher as compared to the others (Fig. 2(B) and (C)). After treatment, the most closely related variables were C15:0, C16:0, C16:1n-9, WC, HC, and WHR (Fig. 2(E) and (F)). The selected key variables were the same as the results of the 118 T2DM patients (Fig. S3†).

\section{Index pattern relating to the onset of AS with T2DM}

Among the 118 T2DM patients, 44 patients developed AS two years later, according to the long-term clinical follow-up. A PLSDA model for the 44 T2DM-AS patients was established (Fig. 3) 
Table 2 The clinical data and FFA concentrations for the T2DM and T2DM-AS patients before and after two years of treatment ${ }^{a}$

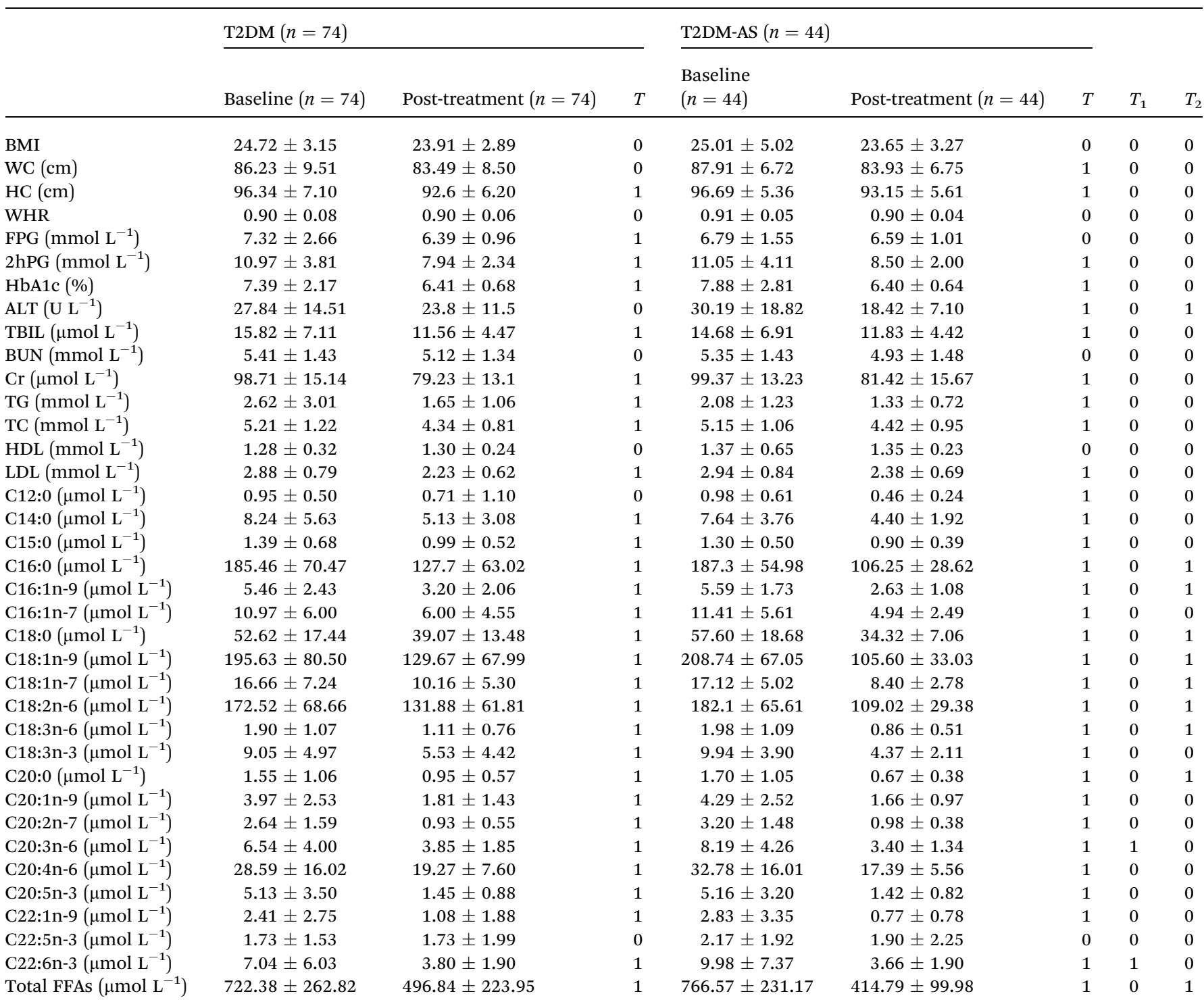

${ }^{a}$ The clinical data and FFA concentrations are presented as mean \pm SD. $T$ is the Mann-Whitney $U$ test results of T2DM or T2DM-AS patients before and after two years of treatment. $T_{1}$ and $T_{2}$ are the results for T2DM and T2DM-AS patients before treatment, T2DM and T2DM-AS patients after two years of treatment, respectively. $p$-value of $<0.05$ was considered statistically significant and the signed $T$ value is " 1 ", otherwise " 0 ".

for the identification of the index pattern relating to the onset of AS with T2DM. Based on the PLS-DA model, the AUC value of 10fold cross-validation was $97.49 \%$. The total correct rate for the 44 T2DM-AS patients was $95.45 \%$ and the correct rates for these patients before and after two years of treatment were 90.91\% and $100 \%$, respectively. On the PLS-DA model with good reliable total correct rate and AUC shown in Fig. S4, $\uparrow \mathrm{Cr}, \mathrm{HbA1c}, \mathrm{C} 20: 2 \mathrm{n}-$ 7, C20:5n-3, and C20:0 were the key indices by coefficient $\beta$. After these five indices were used as input data for the establishment of a new PLS-DA model, the total correct rate and AUC value were $93.18 \%$ and $96.8 \%$, respectively. Of the five indices, HbA1c and C20:0 were different from the index pattern of the treatment of T2DM patients. After two years of treatment, the HbA1c level of T2DM and T2DM-AS both significantly decreased (Table 2). After treatment, C20:0 level decreased, but the level of T2DM was higher than T2DM-AS.
The connection between clinical indices and FFA metabolic profiles was further explored by analyzing the 15 clinical data and 21 FFA concentrations of each of the 44 T2DM-AS patients through CCA. The first pair of canonical variables of metabolite $\left(U_{1}\right)$ and clinical indices $\left(V_{1}\right)$ before and after the two years of treatment of the T2DM-AS patients are shown in Fig. 4. The data show that $R=0.9822$ in Fig. $4(\mathrm{~A})$ and $R=0.9509$ in Fig. 4(D). The coefficients' absolute values of the first pair of canonical variables in the clinical indices and fatty acids of 44 T2DM-AS patients are shown in Fig. 4(B, C, E and F). The coefficients' absolute values of C18:0, C20:2n-7, C22:6n-3, WC, WHR, and ALT were higher than the others before treatment; while the values of C22:6n-3, C16:1n-9, C22:1n-9, WC, HC, and WHR were higher than the others after treatment. We suggest that these variables were closely related to fatty acid metabolism and the clinical indices for T2DM-AS. 
A

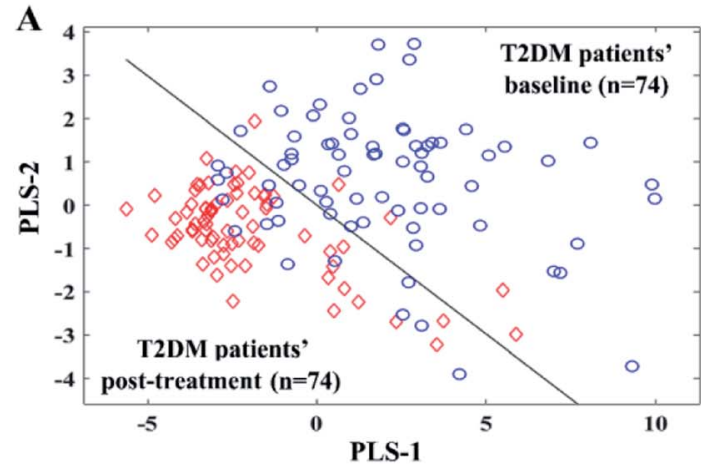

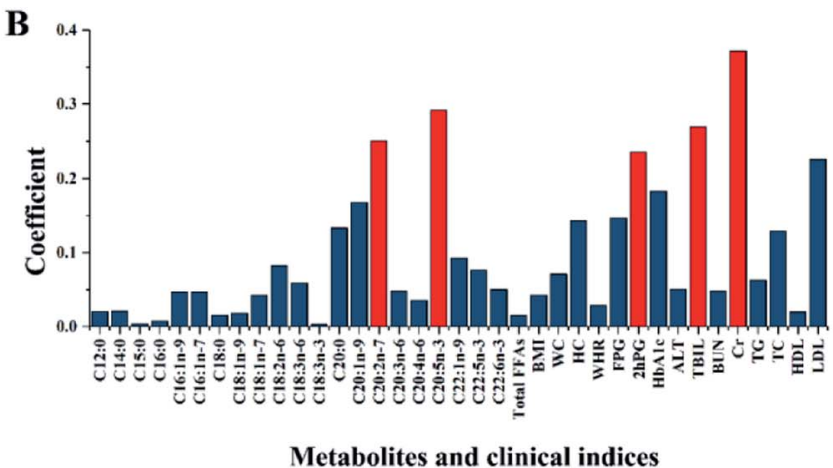

Fig. 1 2D-project plots obtained by PLS-DA for the 74 T2DM patients without AS before and after treatment (A) and the absolute value of coefficient $\beta$ of each variable for discrimination (B). Each projection point in (A) indicates either a baseline (blue) or a post-treatment (red) sample. The AUC value was $96.05 \%$. Correct rates of 10 -fold cross-validation for baseline and post-treatment were $87.84 \%$ and $94.59 \%$, respectively. Cr, TBIL, 2hPG, C20:2n-7, and C20:5n-3 were screened out as key indices.

\section{Discussion}

Diabetes mellitus is a powerful risk factor for cardiovascular disease, and individuals with diabetes have a 2 - to 4 -fold increased risk of developing cardiovascular diseases as compared with those without diabetes. ${ }^{15}$ In this study, 25 male T2DM patients $(25 / 64,39.06 \%)$ and 19 female T2DM patients (19/54, 35.19\%) were complicated with AS after two years. The morbidity of AS for males was higher than in females..$^{29}$ In this study, the incidence of AS in males was higher than that in females. For the 118 T2DM patients, blood sugar, blood pressure, and blood lipids were controlled well after treatment. The development of AC in the 44 T2DM patients was investigated by evaluating 26 lipid metabolic indices ( 21 FFAs, total FFAs, HDL, LDL, TC, and TG), three blood sugar indices (FPG, 2hPG, and HbA1c), four body indices (BMI, WC, HC, and WHR), ALT, TBIL, BUN, and $\mathrm{Cr}$ in these patients before and after treatment. The plasma cholesterol level is a strong predictor of the risk of cardiovascular events both in patients with diabetes and in patients with coronary heart disease..$^{30} \mathrm{HDL}$, LDL, TC, and TG are four important indices to evaluate plasma lipid levels in the clinic. For the T2DM and T2DM-AS patients, HDL,

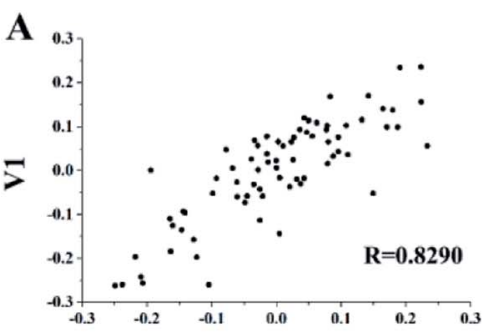

U1

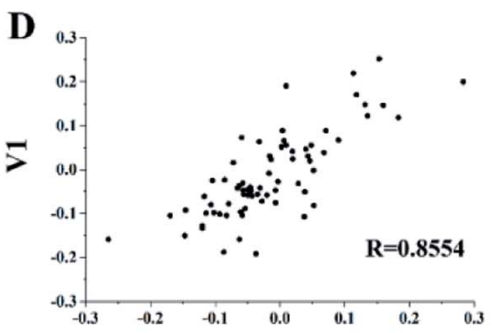

U1
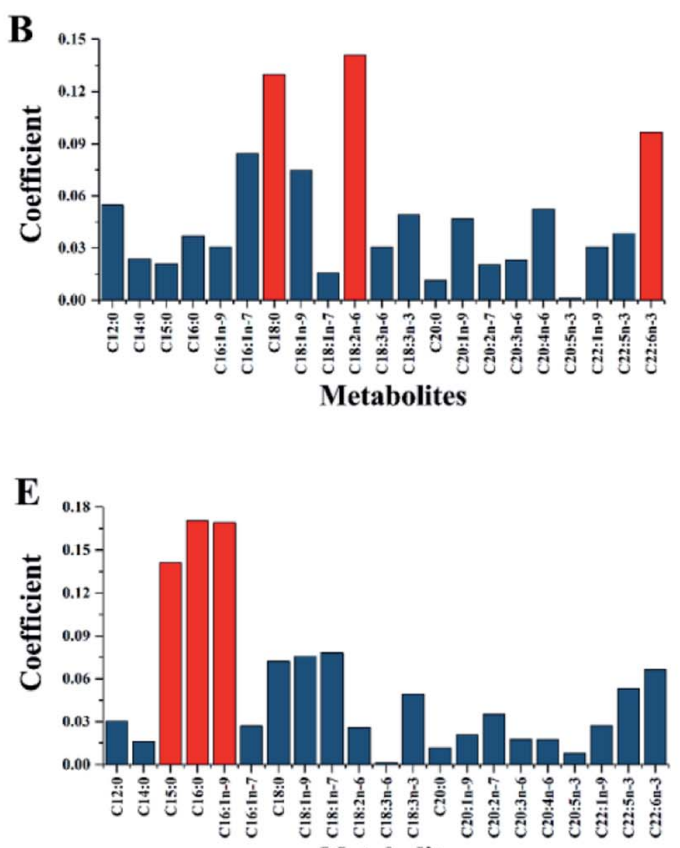
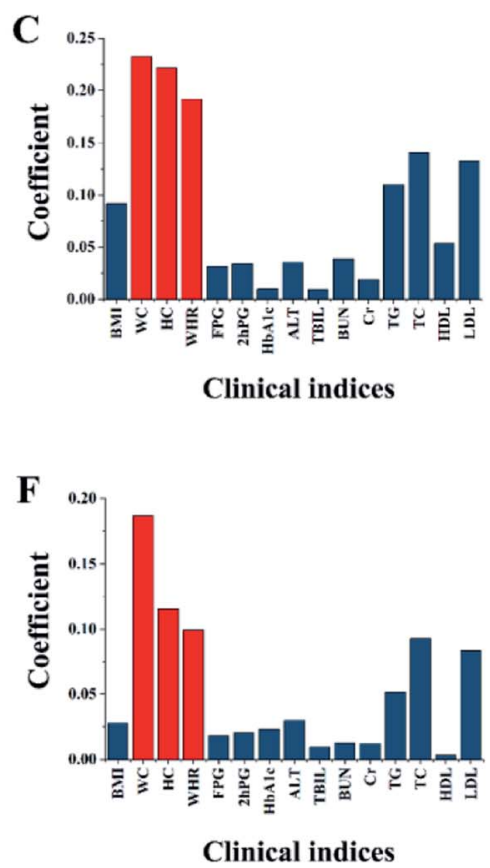

Fig. 2 The results of CCA for the 74 T2DM patients without AS. (A) and (D) show the correlation of the first pair of canonical variables of metabolites $\left(U_{1}\right)$ and clinical indices $\left(V_{1}\right)$ for the 74 T2DM patients before and after two years of treatment. (B) and (E) show the coefficients' absolute values of the first pair of canonical variables in fatty acids of the 74 T2DM patients before and after two years of treatment. (C) and (F) show the coefficients' absolute values for the first pair of canonical variables in the clinical indices of the 74 T2DM patients before and after two years of treatment. $R$ is the correlation coefficient of the corresponding $U_{1}$ and $V_{1}$. 
A

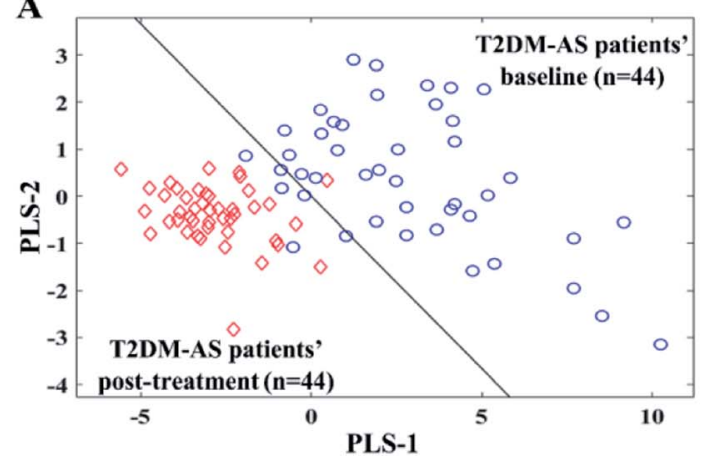

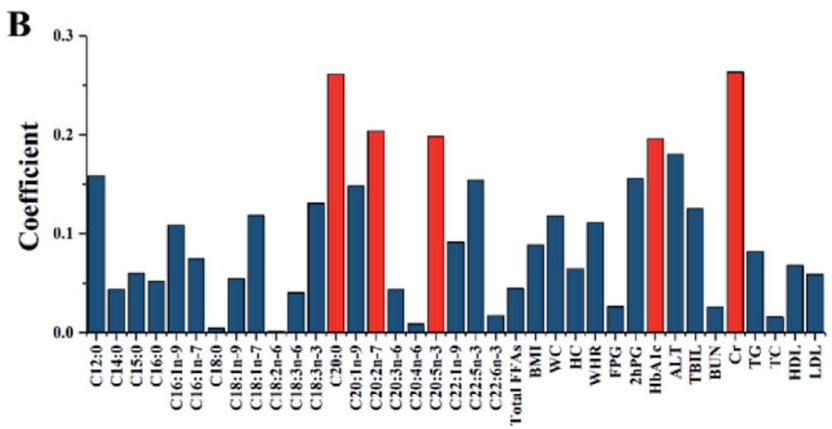

Metabolites and clinical indices

Fig. 3 2D-project plots obtained by PLS-DA for the 44 T2DM-AS patients before and after treatment (A) and the absolute value of coefficient $\beta$ for each variable for the discrimination (B). Each projection point in (A) indicated either a baseline (blue) or a post-treatment (red) sample. The AUC value was $97.49 \%$. The correct rates of 10 -fold cross-validation for baseline and post-treatment were $90.91 \%$ and $100 \%$, respectively. Cr, HbA1c, C20:2n-7, C20:5n-3, and C20:0 were screened out as key indices.

LDL, TC and TG had no significant differences. The control of total FFAs was also good.

To further obtain useful information from the data, multivariate statistical analysis was employed. In the combined results of PLS-DA and CCA, we suggested that C20:2n-7, C20:5n3 , WC, and WHR are four key factors for the treatment of T2DM, and C20:0, C22:6n-3, HbA1c, WC, and WHR are key factors for the onset of AS with T2DM.

The clinical indices WC and WHR reflect the condition of systemic fat accumulation. These indices were selected not only for the treatment of T2DM but also for the onset of AS. For
T2DM-AS, WC decreased notably, but the change in WHR was small. The control of WHR might be more important than that for WC in the prevention of AS onset. Considering that glucose and lipid metabolism disorders may have influenced the occurrence of AS, we analyzed FPS, 2hPG, HbA1c, TG, TC, HDL and LDL levels between T2DM and T2DM-AS group, and found that the above parameters were comparable in the two groups at baseline and after treatment. However, $37.29 \%$ patients were complicated with AS two years later, suggesting that comprehensive managements that make classical risk factors such as blood glucose and blood lipids meet the criteria cannot

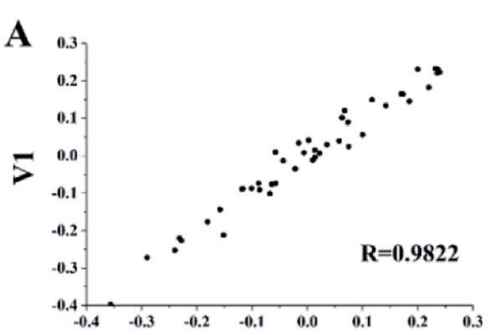

U1

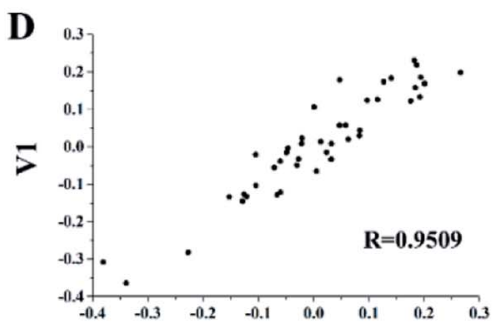

U1

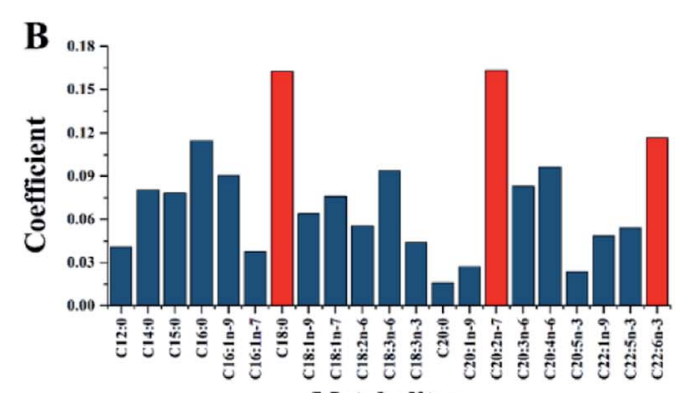

Metabolites

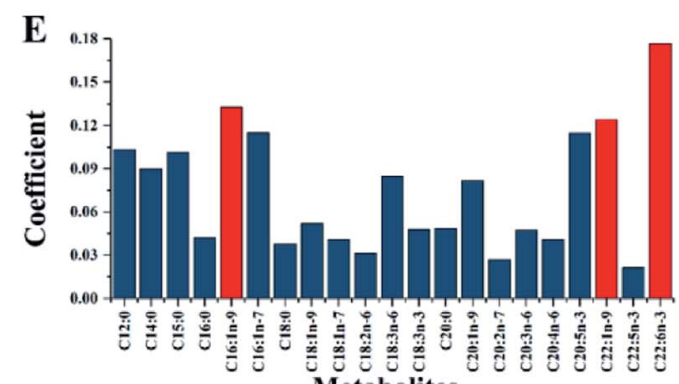

Metabolites

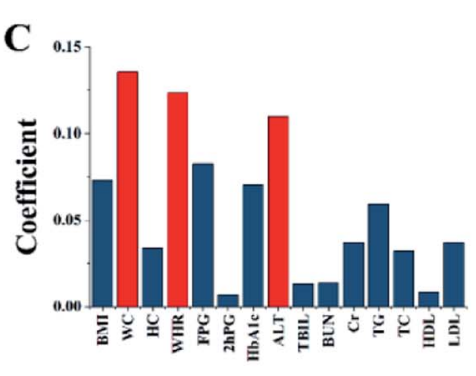

Clinical indices

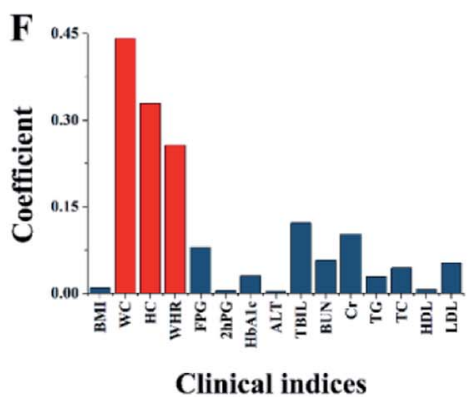

Fig. 4 The results of the canonical correlation analysis for the 44 T2DM-AS patients: (A) and (D) show the correlation of the first pair of canonical variables of metabolites $\left(U_{1}\right)$ and clinical indices $\left(V_{1}\right)$ for the 44 T2DM-AS patients before and after two years of treatment. (B) and $(E)$ show the coefficients' absolute values of the first pair of canonical variables in fatty acids of the 44 T2DM-AS patients before and after two years of treatment. (C) and (F) showed the coefficients' absolute values of the first pair of canonical variables in the clinical indices of 44 T2DM-AS patients before and after two years of treatment. $R$ was the correlation coefficient of corresponding $U_{1}$ and $V_{1}$. 
completely prevent the development of AS. Improved glycemic control is known to slow the onset and progression of microvascular complications, but it had little effect on macrovascular disease. At the same time, the correction of lipoprotein abnormalities will lead to a decrease in coronary-artery disease. ${ }^{\mathbf{1 4}}$

Polyunsaturated fatty acids improve insulin resistance and are potentially protective against T2DM, particularly $n-3$ fatty acids, which may increase HDL cholesterol levels and might have the following effects: anti-inflammatory, antiatherosclerotic, and lowering blood pressure and triglycerides. ${ }^{31,32}$ C20:5n-3 (EPA) was screened as a key factor for T2DM treatment in this study. It is formed from C18:3n-3 with the help of fatty acid elongase and desaturase, and it is a precursor of anti-inflammatory eicosanoids. Reportedly, eight weeks supplementation of EPA had beneficial effects on methionine and cysteine. ${ }^{33}$ These effects of EPA may contribute to cardioprotection in patients with obesity and metabolic syndrome. ${ }^{34}$ In this study, the level of total FFAs for T2DM patients decreased by $31.22 \%$ (45.89\% for T2DM-AS), from $722.38 \mu \mathrm{mol} \mathrm{L}{ }^{-1}$ to $496.84 \mu \mathrm{mol} \mathrm{L}{ }^{-1}$. The level of $\mathrm{C} 20: 5 \mathrm{n}-3$ decreased by $71.73 \%$ (72.48\% for T2DM-AS), from $5.13 \mu \mathrm{mol}$ $\mathrm{L}^{-1}$ to $1.45 \mu \mathrm{mol} \mathrm{L} \mathrm{L}^{-1}$. C20:2n-7 is a type of eicosadienoic acid, which can lower risk of inflammation. ${ }^{35}$

C22:6n-3 (DHA) has a positive effect on diseases such as hypertension, arthritis, AS, depression, adult-onset diabetes mellitus, myocardial infarction, thrombosis, and some cancers $^{36}$ and C20:3n-6 (DGLA) has an anti-atherogenic effect. ${ }^{37}$ In this study, T2DM-AS patients showed higher levels of DHA and DGLA two years before the treatment than T2DM patients, which is in conflict with some reports. ${ }^{38-40}$ However, the level of DHA (9.98-3.66 $\left.\mu \mathrm{mol} \mathrm{L}{ }^{-1}\right)$ and DGLA (8.19-3.40 $\mu \mathrm{mol} \mathrm{L}^{-1}$ ) for T2DM-AS patients decreased more obviously than T2DM patients (DHA, 7.04-3.8 $\mu \mathrm{mol} \mathrm{L}{ }^{-1}$, DGLA, 6.54-3.85 $\mu \mathrm{mol} \mathrm{L}^{-1}$ ). There is no exact knowledge at this stage, but Ryo Ito et al. found that AA, DHA, and DGLA were significantly higher in patients with dyslipidemia than those with non-dyslipidemia, ${ }^{34}$ and Yuriko Abe found high levels of DGLA in the blood of obese children. $^{41}$

Most of the high mortality risk of T2DM is attributed to macrovascular AS disease. ${ }^{\mathbf{1 4}}$ Thus, it has been recommended that medical management to decrease cardiovascular risk should start when type 2 diabetes mellitus is diagnosed. The results of this study suggested that the simple regulation of these existing clinical indices does not control the development of AS well, and it is also difficult to predict the prognosis of the patients. Plasma fatty acid metabolic profiling research can provide rich metabolite information and useful supplements for existing clinical indices to predict the development of patients' disease more effectively and optimize treatment plans such as the addition of some lipid species to improve the risk prediction of cardiovascular events and death. ${ }^{42}$

\section{Conclusions}

Current clinical indices cannot provide a complete view of an individual's risk for future cardiovascular events, especially T2DM patients. The combination of metabolomics research may improve the current risk assessment models. In this study, the alterations of the clinical indices and FFAs profiles were evaluated in 118 T2DM patients before and after two years of treatment. Through advanced analytical technology and efficient chemometric methods, some interesting results were obtained. C20:2n-7, C20:5n-3, WC, and WHR were the key factors for the treatment of T2DM, and C20:0, C22:6n-3, HbA1c, WC, and WHR were the key factors for the onset of AS with T2DM. These new findings provide information for the discriminatory risk assessment of atherosclerosis in T2DM patients. It might be important to guide the clinical intervention of T2DM.

\section{Conflicts of interest}

The authors declare no competing financial interests.

\section{Abbreviations of clinical indices}

$\begin{array}{ll}\text { BMI } & \text { Body mass index } \\ \text { WC } & \text { Waist circumference } \\ \text { HC } & \text { Hip circumference } \\ \text { WHR } & \text { Waist hip ratio } \\ \text { FPG } & \text { Fasting plasma glucose } \\ \text { 2hPG } & \text { 2 hour postprandial plasma glucose } \\ \text { HbA1c } & \text { Glycosylated hemoglobin } \\ \text { ALT } & \text { Alanine transaminase } \\ \text { TBIL } & \text { Total bilirubin } \\ \text { BUN } & \text { Blood urea nitrogen } \\ \text { Cr } & \text { Creatinine } \\ \text { TG } & \text { Triglyceride } \\ \text { TC } & \text { Total cholesterol } \\ \text { HDL } & \text { High density lipoprotein } \\ \text { LDL } & \text { Low density lipoprotein }\end{array}$

\section{Acknowledgements}

This work is financially supported by the National Natural Science Foundation of P. R. China (Grant No. 21775058, 21465016 and 11761041).

\section{References}

1 B. Hocher and J. Adamski, Nat. Rev. Nephrol., 2017, 13(5), 269-284.

2 B. V. Ravenzwaay, M. Herold, H. Kamp, et al., Mutat. Res., 2012, 746(2), 144-150.

3 H. Wu and W. Wang, Environ. Toxicol. Chem., 2015, 30(4), 806-812.

4 P. Würtz, Q. Wang, P. Soininen, et al., J. Am. Coll. Cardiol., 2016, 67(10), 1200-1210.

5 Y. Chiba, T. Yamakawa, H. Tsuchiya, et al., J. Clin. Med. Res., 2018, 10(8), 648-656.

6 J. Y. Hwang, H. J. Lee, J. G. Min, et al., Sci. Rep., 2016, 6, 33043.

7 F. B. Hu, T. Y. Li, G. A. Colditz, et al., J. Am. Med. Assoc., 2003, 289(14), 1785-1791. 
8 A. Pan, Y. Wang, M. Talaei, et al., Lancet Diabetes Endocrinol., 2015, 3(12), 958-967.

9 World Health Organization, Global report on diabetes, World Health Organization, Geneva, 2016.

10 A. Keech, Atheroscler. Suppl., 2006, 7(3), 342.

11 R. B. Goldberg, D. M. Kendall, M. A. Deeg, et al., Diabetes Care, 2005, 28(12), 2985-2986.

12 Z. Nachum, N. Zafran, R. Salim, et al., Diabetes Care, 2017, 40(3), 332-337.

13 J. Ma, L. Y. Liu, P. H. Wu, et al., J. Diabetes Res., 2014, 294017.

14 G. Steiner, A. Hamsten, J. Hosking, et al., Lancet, 2001, 357(9260), 905-910.

15 M. Laakso, T. Ronnemaa, K. Pyorala, et al., Diabetes Care, 1988, 11(6), 449-463.

16 T. W. Gress, F. J. Nieto, E. Shahar, et al., N. Engl. J. Med., 2000, 343(13), 905-912.

17 H. G. McGill, J. P. Strong, R. E. Tracy, et al., Arterioscler., Thromb., Vasc. Biol., 1995, 15(12), 2222-2228.

18 S. M. Grundy, H. Arai, P. Barter, et al., Atherosclerosis, 2014, 8(1), 29-60.

19 T. J. Chahil and H. N. Ginsberg, Endocrinol. Metab. Clin. North Am., 2006, 35(3), 491-510.

20 R. Scott, R. O'Brien, G. Fulcher, et al., Diabetes Care, 2009, 32(3), 493-498.

21 J. M. Nie and H. F. Li, Exp. Ther. Med., 2017, 14(3), 25212526.

22 Y. Q. Bao, T. Zhao, X. Y. Wang, et al., J. Proteome Res., 2009, 8(4), 1623-1630.

23 Y. Xiao, X. Y. Xiao, A. Xu, et al., J. Diabetes Complicat., 2018, 32(12), 1100-1104.

24 E. Expert Panel on Detection, Treatment of High Blood Cholesterol in A, J. Am. Med. Assoc., 2001, 285(19), 24862497.

25 Y. Xiao, A. Xu, X. Y. Hui, et al., PLoS One, 2013, 8(6), e66607.
26 L. Z. Yi, J. He, Y. Z. Liang, et al., Chem. Phys. Lipids, 2007, 150(2), 204-216.

27 L. Z. Yi, J. He, Y. Z. Liang, et al., FEBS Lett., 2006, 580(30), 6837-6845.

28 L. Z. Yi, N. P. Dong, Y. H. Yun, et al., Anal. Chim. Acta, 2016, 914, 17-34.

29 G. C. Flora, A. B. Baker, R. B. Loewenson, et al., Circulation, 1968, 38(5), 859-869.

30 P. L. D. Luz, D. Favarato, J. R. F. N. Junior, et al., Clinics, 2008, 63(4), 427-432.

31 M. G. Jacobo-Cejudo, R. Valdés-Ramos and A. L. GuadarramaLópez, Nutrients, 2017, 9, 573-583.

32 K. Ide, M. Koshizaka, H. Tokuyama, et al., Lipids Health Dis., 2018, 17(1), 51.

33 M. H. Golzari, M. H. Javanbakht, E. Ghaedi, et al., Diabetes Metab. Syndr., 2018, 12(3), 411-415.

34 R. Ito, N. Satoh-Asahara, H. Yamakage, et al., J. Atheroscler. Thromb., 2014, 21(3), 248-260.

35 Y. S. Huang, W. C. Huang, C. W. Li, et al., Mol. Cell. Biochem., 2011, 358(1-2), 85-94.

36 L. A. Horrocks and Y. K. Yeo, Pharmacol. Res., 1999, 40(3), 211-225.

37 S. Takai, D. Jin, H. Kawashima, et al., J. Atheroscler. Thromb., 2009, 16, 480-489.

38 L. Liu, Q. Hu, H. Wu, et al., J. Nutr. Biochem., 2016, 32, 171180.

39 D. S. Kelley and Y. Adkins, Proc. Nutr. Soc., 2012, 71(02), 322331.

40 S. H. Ahmed, N. Kaoubaa, W. Kharroubi, et al., Lipids Health Dis., 2017, 16(1), 154.

41 Y. Abe, T. Okada, H. Iguchi, et al., J. Atheroscler. Thromb., 2012, 19(12), 1102.

42 Z. H. Alshehry, P. A. Mundra, C. K. Barlow, et al., Circulation, 2016, 134, 1637-1650. 